Norma Helen Medina 1

Vera Lúcia Gattás 2

Gilberto L. Anjos 3

Conchita Montuori 1

Rosana Maura Gentil 1

\section{Prevalência de tracoma em pré-escolares e escolares no Município de Botucatu, São Paulo, Brasil, 1992}

\author{
Trachoma prevalence in preschoolers \\ and schoolchildren in Botucatu, São Paulo \\ State, Brazil, 1992
}

1 Serviço de Oftalmologia Sanitária, Centro de Vigilância Epidemiológica Professsor Alexandre Vranjac, Secretaria de Estado da Saúde de São Paulo. Av. Dr. Arnaldo 351, São Paulo, SP 01246-902, Brasil.

2 Instituto de Saúde, Secretaria de Estado da Saúde de São Paulo. Rua Santo Antonio 590, São Paulo, SP 01314-000, Brasil.

3 Diretoria Regional de Saúde de Botucatu, Secretaria de Estado da Saúde de São Paulo. Rua General Telles 251, Botucatu, SP 18603-710, Brasil.

\begin{abstract}
Trachoma dispensaries were created in the State of São Paulo in 1938, when the incidence of trachoma was 10.9\% in Botucatu. During the 1970s trachoma ceased to pose a public health problem. The disease reappeared in the 1980s, leading the São Paulo State Health Department to establish a control program as new cases were being detected in various parts of the State, including Botucatu. This study aimed to determine the prevalence of trachoma in preschoolers and schoolchildren and to implement a program to control the disease in Botucatu. A cluster sample of children were submitted to external ocular examination to detect signs of trachoma according to WHO criteria. Trachoma prevalence was 11.9\% among the 1,393 children examined. All cases were treated with 1.0\% tetracycline ointment and followed up periodically. Loss to follow-up increased from $1.6 \%$ for the first return visit to $62.4 \%$ for the last visit. The results showed the need to investigate the causes of non-compliance with the control program and the impact and effectiveness of treatment.
\end{abstract}

Key words Trachoma; School Health; Epidemiologic Surveillance; Prevalence

Resumo Em 1938, foram criados os Dispensários de Tracoma em São Paulo, entre eles, o de Botucatu, onde a incidência de tracoma era de 10,9\%. Na década de 70, o tracoma passou a não ser mais um problema de saúde pública. A doença reapareceu no início dos anos 80, levando a Secretaria de Estado da Saúde de São Paulo (SES-SP), a implantar um programa de controle, detectando-se casos novos em diversos locais, inclusive em Botucatu. Realizou-se esta investigação com o objetivo de conhecer a prevalência do tracoma em escolares e pré-escolares da rede de ensino, e implantar um programa de controle da doença em Botucatu, São Paulo. As crianças foram submetidas a exame ocular externo para detectar sinais de tracoma, seguindo os critérios da Organização Mundial da Saúde. Foram examinadas 1.393 crianças, encontrando-se uma prevalência de tracoma de 11,9\%. Todos os casos foram tratados com pomada de tetraciclina a 1,0\% e controlados. Durante os controles, observou-se um aumento da freqüência de faltosos de $1,6 \%$ no primeiro retorno a $62,4 \%$ para último retorno. Os resultados revelaram a necessidade de se conhecer melhor as causas da não-aderência ao programa e o seu impacto na efetividade do tratamento do tracoma.

Palavras-chave Tracoma; Saúde Escolar; Vigilância Epidemiológica; Prevalência 


\section{Introdução}

Os primeiros casos de tracoma que se tem referência no Estado de São Paulo datam de 1890, originados da imigração estrangeira. O tracoma disseminou-se rapidamente para o oeste do estado, acometendo principalmente os colonos da região. As estatísticas de 1907 indicavam para o Estado de São Paulo, um índice de detecção de casos de tracoma de 27,56\% (Melo-Oliveira, 1927).

Em 1938, foi criado o Instituto do Tracoma do Estado de São Paulo, organizando serviços de profilaxia, com caráter permanente, através de sistema de unidades especializadas, anexas aos centros de saúde: os dispensários de tracoma (Toledo, 1944). Com base em dados coletados desde 1938, de clínicas especializadas, dispensários e postos rurais de tracoma entre outros, em 1944, foram determinadas sete zonas de incidência de tracoma, e o Município de Botucatu fazia parte da terceira zona, com uma incidência de 10,1\% de tracoma em escolares (Toledo, 1944).

Houve um declínio gradual da prevalência da doença a partir da década de 50, principalmente devido à melhoria das condições sócioeconômicas do país (Luna, 1993). Com a reforma administrativa da Secretaria Estadual da Saúde de São Paulo (SES-SP), em 1968, o Instituto do Tracoma e a rede de dispensários foram extintos e em 1969, transformaram-se no Serviço de Oftalmologia Sanitária, que continuou divulgando informações sobre a ocorrência da doença entre os dados de atendimento oftalmológico. Nesse período, verifica-se um declínio na proporção de casos de tracoma em relação ao total dos atendimentos oftalmológicos, o que levou-se a acreditar que a doença estava em processo de erradicação. A partir de 1975, não se publica nenhum dado de ocorrência de tracoma no Estado. Com a implantação do Sistema de Vigilância Epidemiológica (SVE) em 1978, verificou-se que o tracoma deixou de figurar no rol das doenças de notificação compulsória (Luna, 1993).

Contudo, em 1982, novos casos foram detectados em pré-escolares na cidade de Bebedouro, alcançando 749 casos entre 1984 e 1985 (Luna et al., 1987). Desde 1989, a SES-SP vem implantando um programa para o controle do tracoma com uma programação ampliada de treinamentos. Como reflexo de um maior número de profissionais capacitados, verificou-se a organização de programas de controle do tracoma em vários municípios, com a realização de busca ativa e uma explosão no número do casos notificados ao SVE do Estado, chegando a cerca de 10 mil em 1991 (SES-SP, 1993).
O tracoma volta a ser considerado oficialmente, uma doença de notificação compulsória para o Estado de São Paulo em 1992 (São Paulo, 1994).

Em 1991, foram notificados três casos ao Escritório Regional de Saúde de Botucatu, todos procedentes desse município, e a partir de então foram desencadeadas as ações de vigilância epidemiológica para o tracoma, que incluíram busca ativa de casos e controle de comunicantes, tratamento e acompanhamento.

Os três casos notificados no ano anterior, foram reexaminados no início de junho de 1992, observando-se que, apesar de terem recebido algum tipo de tratamento, mantinham alterações clínicas compatíveis com inflamação tracomatosa folicular (TF), sendo detectados outros casos entre os comunicantes intra-domiciliares.

Dando continuidade às ações, realizou-se nova busca ativa na escola dos primeiros casos, detectando-se mais 18 ocorrências de TF. Realizou-se coleta de material conjuntival em todos os casos encontrados, que confirmaram a presença de Chlamydia trachomatis.

Diante da situação, optou-se por uma investigação da prevalência da doença no município, para se conhecer a magnitude do problema e assim subsidiar suas ações de vigilância epidemiológica.

\section{Material e método}

O Município de Botucatu localiza-se a $247 \mathrm{~km}$ da capital do Estado de São Paulo, e tinha uma população de 90.716 habitantes em 1991 (IBGE, 1994).

A população estudada era composta de préescolares (4 a 6 anos, 30\%) e escolares do " $\mathrm{Ci}$ clo Básico" (1a e 2ạ séries, 70\%).

Para a seleção da amostra, utilizou-se listagens de classes e número de crianças matriculadas em todas as pré-escolas e escolas da rede oficial pública e privada do município de Botucatu, incluindo-se as da zona rural, que foram fornecidas pela Coordenadoria de Educação e Cultura Municipal, Delegacia Regional de Ensino do Estado e diretamente pelas escolas. Dessa maneira, todas as crianças institucionalizadas tiveram a mesma probabilidade de participar da amostra.

Para a execução do estudo, foi utilizado o processo de amostragem por conglomerados, em um único estágio. A amostra foi selecionada por sorteio sistemático, tendo como unidade amostral a sala de aula.

O tamanho da amostra foi determinado, considerando uma prevalência de $6,7 \%$ de tra- 
coma em crianças sob todas as formas (Luna et al., 1992), aceitando-se um erro amostral de 5\% e o intervalo de confiança de 0,0161 (Dean et al., 1994). O número obtido foi multiplicado por dois, com objetivo de corrigir o efeito do desenho, visto que o estudo foi feito por conglomerado. A amostra foi acrescida em $20,0 \%$, dado que estudos anteriores demostraram a ocorrência de aproximadamente $19,0 \%$ de perda com faltosos e desistentes, apesar das repetidas visitas às escolas (Medina et al., 1994).

\section{Exame oftalmológico}

Foi realizado exame ocular externo, utilizando lupa de 2,0 aumentos, com iluminação natural ou artificial com lanterna, em todos os indivíduos da amostra.

Os casos de tracoma foram diagnosticados clinicamente, de acordo com o sistema adotado pela Organização Mundial da Saúde (OMS) (Thylefors et al., 1987). Foram considerados casos de tracoma os indivíduos que apresentavam um ou mais dos seguintes sinais:

- Tracoma inflamatório folicular (TF): presença de 5 ou mais folículos, com pelo menos 0,5 mm de diâmetro, na área delimitada da conjuntiva tarsal superior.

- Tracoma inflamatório intenso (TI): espessamento inflamatório acentuado da conjuntiva, suficiente para obscurecer em mais da metade dos vasos tarsais profundos.

- Tracoma cicatricial (TS): presença de cicatrizes na conjuntiva tarsal superior.

- Triquíase tracomatosa (TT): presença de pelo menos um cílio invertido tocando o globo ocular ou removido recentemente.

- Opacificação corneana (CO): opacidade na córnea que atinge a região da borda pupilar, causando uma diminuição da acuidade visual.

Foi coletado raspado conjuntival para realização de exame laboratorial por imunofluorescência direta para C. trachomatis, com o objetivo de confirmar a presença da circulação da bactéria nos casos detectados.

\section{Tratamento}

Todos os casos de tracoma inflamatório (TF/TI) foram tratados com pomada oftálmica de tetraciclina a $1,0 \%$, duas vezes ao dia, durante seis semanas, conforme preconização da SESSP, e também receberam orientações quanto ao tratamento e cuidados de higiene (SES-SP, 1993). Quando a prevalência alcançou 20,0\% na classe, foi realizado tratamento em massa segundo critério estabelecido pela OMS (Thylefors et al., 1987).

\section{Controle dos casos}

Os casos de tracoma diagnosticados foram notificados ao SVE de São Paulo, e controlados pelo Centro de Saúde local.

O controle dos casos de tracoma inflamatório (TF/TI), foi realizado em quatro atendimentos com intervalo de seis semanas cada, por um período aproximado de seis meses.

No retorno do primeiro atendimento, o caso que não apresentava sinais de tracoma inflamatório ativo, isto é, ausência total de folículos e hiperemia conjuntival, foi considerado em alta clínica, sendo suspensa a medicação preconizada, devendo retornar por mais três vezes ao atendimento de controle para receber alta curado.

O indivíduo que após ter recebido alta clíni$c a$, retornasse com sinais de tracoma em um dos próximos atendimentos de controle, era considerado uma recidiva, recebendo novamente medicação específica.

O caso que não compareceu em um dos retornos de atendimentos do controle do tratamento foi considerado faltoso ao agendamento.

\section{Resultados}

Foram examinados 1.393 pré-escolares e escolares da rede de ensino pública e privada do Município de Botucatu, com 26,3\% de perda na amostra, sendo semelhante a distribuição por sexo e faixa etária da amostra e das perdas.

A prevalência de tracoma total na amostra foi de $11,9 \%$, sendo $11,6 \%$ de TF, $0,2 \%$ de tracoma inflamatório intenso (TF/TI) e $0,1 \%$ de tracoma inflamatório associado à forma cicatricial (TF/TI/TS) (Figura 1).

A prevalência de tracoma por sexo foi 11,89\% em ambos os sexos.

A distribuição dos casos de tracoma segundo faixa etária encontra-se na Tabela 1 , onde observa-se uma prevalência de $25,0 \%$ entre as crianças de quatro anos e de $23,7 \%$ entre as de cinco. Nas demais idades, a distribuição apresentou percentuais de $8,1 \%$ para as crianças de 9 e 10 anos, a 13,2\% para as de 11 ou mais.

Os casos estavam distribuídos desigualmente pelas escolas examinadas, sendo que a prevalência de tracoma total variou de zero até $41,9 \%$. Porém, quando os mesmos foram mapeados por endereço no município, a distribuição geográfica mostrou-se homogênea.

Foi confirmada, laboratorialmente, a presença de C. trachomatis nos casos detectados.

No primeiro atendimento de controle de tratamento, $32,2 \%$ estavam usando corretamente 
a medicação, e somente $6,4 \%$ dos casos apresentaram alta clínica. Com base nos dados coletados, evidenciou-se que houve, provavelmente, falha na orientação sobre o tratamento e controle, pois $53,3 \%$ dos casos estavam usando irregularmente a pomada e $14,5 \%$ não usavam a medicação.

A freqüência de faltosos aos controles foi de $1,6 \%$ no primeiro retorno e alcançou $62,4 \%$ no último controle.

Figura 1

Prevalência de tracoma em pré-escolares e escolares.

Município de Botucatu, São Paulo, Brasil, 1992.

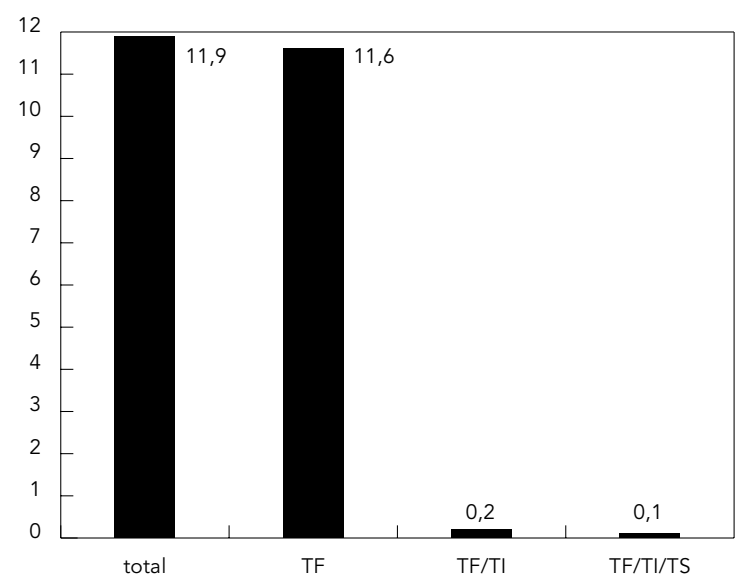

$\mathrm{TF}$ = tracoma inflamatório folicular; $\mathrm{TI}$ = tracoma inflamatório intenso; TS $=$ tracoma cicatricial.

\section{Discussão}

A prevalência de tracoma inflamatório (TF/TI) de $11,9 \%$, foi maior do que a encontrada em outros municípios do Estado de São Paulo, como em Guaraci de 9,6\%, em Cajobi 6,7\%, Olímpia 5,4\% e em Franco da Rocha 1,5\% (Medina et al., 1992, 1994). Porém, essa prevalência é menor quando comparada com a de outros municípios do Brasil, como Miraselva, Paraná $(51,7 \%)$; Porecatu, Paraná $(20,4 \%)$; Mocambo, Ceará $(22,2 \%)$ e em áreas hiperendêmicas de países em desenvolvimento (Moreira et al., 1993; Scarpi et al., 1989; Tielsh et al., 1988).

Não houve diferença na distribuição de tracoma entre o sexo feminino e o masculino, o que está de acordo com a literatura nacional (Medina et al., 1994; Nóbrega et al., 1993).

A prevalência de TF de $25,0 \%$ na faixa etária de quatro anos e de $23,7 \%$ na de cinco, é semelhante ao estudo realizado nas cidades de Olímpia, Guaraci e Cajobi, São Paulo, onde foi encontrada uma prevalência de $24,0 \%$ na faixa etária de quatro anos e 20,0\% na de cinco (Medina et al., 1992). Porém, são mais altas quando comparadas às de outros estudos realizados no Estado de São Paulo, que encontraram prevalências menores do que 10,0\% em Franco da Rocha, Francisco Morato e Bebedouro (Luna et al., 1992; Medina et al., 1994). Recomenda-se que o programa de controle deva priorizar os pré-escolares, mesmo sendo a prevalência nas outras faixas etárias também alta.

A distribuição dos casos de tracoma segundo local de residência foi homogênea por todo o município, diferentemente de Bebedouro São Paulo, onde a prevalência era maior na periferia da cidade (Luna et al., 1992).

Tabela 1

Número de casos e prevalência de tracoma total, segundo faixa etária, em uma amostra de pré-escolares e escolares. Município de Botucatu, São Paulo, Brasil, 1992.

\begin{tabular}{|c|c|c|c|c|c|c|c|}
\hline \multirow[t]{2}{*}{ Idade (anos) } & \multicolumn{2}{|c|}{ Normal } & \multicolumn{2}{|c|}{ Tracoma } & \multirow{2}{*}{$\begin{array}{c}\text { Intervalo de } \\
\text { confiança } 95 \%\end{array}$} & \multicolumn{2}{|c|}{ Total } \\
\hline & $\mathrm{n}$ & $\%$ & $n$ & $\%$ & & $\mathrm{n}$ & $\%$ \\
\hline 4 & 12 & 75,0 & 4 & 25,0 & $8,3-52,6$ & 16 & 100,0 \\
\hline 5 & 45 & 76,3 & 14 & 23,7 & $14,0-36,9$ & 59 & 100,0 \\
\hline 6 & 247 & 91,1 & 24 & 8,9 & $5,9-13,1$ & 271 & 100,0 \\
\hline 7 & 285 & 87,2 & 42 & 12,8 & $9,5-17,1$ & 327 & 100,0 \\
\hline 8 & 353 & 86,9 & 53 & 13,1 & $10,0-16,8$ & 406 & 100,0 \\
\hline 9 & 158 & 91,9 & 14 & 8,1 & $4,7-13,6$ & 172 & 100,0 \\
\hline 10 & 68 & 91,9 & 6 & 8,1 & $3,3-17,4$ & 74 & 100,0 \\
\hline $11 e+$ & 59 & 86,8 & 9 & 13,2 & $6,6-24,1$ & 68 & 100,0 \\
\hline Total & 1.227 & 88,1 & 166 & 11,9 & $10,3-13,8$ & 1.393 & 100,0 \\
\hline
\end{tabular}


Esses dados demonstram que o tracoma nesta amostra de crianças, apesar de estar apresentando uma prevalência de tracoma inflamatório maior do que a de outros municípios de São Paulo, não deve estar ocorrendo grande número de reinfecções e quadros mais graves, pois só foi encontrado um caso com cicatrizes conjuntivais.

Durante o seguimento dos casos no Centro de Saúde, observou-se um aumento progressivo do número de faltosos alcançando mais de $50 \%$. Em trabalho de acompanhamento dos casos de tracoma em uma instituição de ensino na cidade de São Paulo, verificou-se também um aumento do número de faltosos de $48 \%$ (Medina et al., 1998).

Realizar os controles na instituição onde estão os casos é uma forma de controlar essa variável limitante, visto que muitos têm dificuldade de acesso ao serviço de saúde.

Um outro motivo da baixa aderência ao tratamento e aos controles é a dificuldade da administração de pomada oftálmica por longo tempo.

Os resultados revelaram a necessidade de se conhecer melhor as causas da não-aderên- cia ao programa estabelecido pelos serviços de saúde e, conseqüentemente, o seu impacto na efetividade do tratamento do tracoma.

O tracoma por ser uma doença crônica recidivante, seu acompanhamento é longo e as ações de controle dos casos necessitam ter continuidade. Para alcançar esse objetivo, é importante um trabalho persistente de educação em saúde para conscientizar a população da importância do tratamento e seu controle.

A utilização de inquéritos por amostragem em pré-escolares e escolares, tem se mostrado efetiva para a detecção de casos de tracoma em locais onde quase a totalidade das crianças está freqüentando instituições. Entretanto, a estratégia de controle dos casos encontrados só será efetiva se houver uma equipe de saúde engajada ao programa e com condições de trabalho, a fim de realizar todas as atividades de seguimento dos casos, inclusive visitas domiciliares aos faltosos.

Recomenda-se a implementação do Programa de Vigilância Epidemiológica do Tracoma, com capacitação de profissionais das unidades de saúde do município.

\section{Referências}

DEAN, A. G.; DEAN, J. A.; COULOMBIER, D.; BRENDEL, K. A.; SMITH, D. C.; BURTON, A. H.; DICKER, R. C.; SULLIVAN, K.; FAGAN, R. F. \& ARNER, T. G., 1994. Epi Info, Version 6.4: A Word Processing, Database and Statistics Program for Epidemiology on Microcomputers. Atlanta: Centers for Disease Control and Prevention.

IBGE (Fundação Instituto Brasileiro de Geografia e Estatística), 1994. Censo Demográfico, 1991. v. 21. São Paulo. Rio de Janeiro: IBGE.

LUNA, E. J. A., 1993. A Epidemiologia do Tracoma no Estado de São Paulo. Dissertação de Mestrado, Campinas: Faculdade de Ciências Médicas, Universidade Estadual de Campinas.

LUNA, E. J. A.; MEDINA, N. H. \& OLIVEIRA, M. B., 1987. Vigilância epidemiológica do tracoma no Estado de São Paulo. Arquivos Brasileiros de Oftalmologia, 50:70-79.

LUNA, E. J. A.; MEDINA, N. H.; OLIVEIRA, M. B.; BARROS, O. M.; VRANJAC, A.; MELLES, H. H. B.; WEST, S. \& TAYLOR, H. R., 1992. Epidemiology of trachoma in Bebedouro State of São Paulo, Brazil: Prevalence and risk factors. International Journal of Epidemiology, 21:169-177. 
MEDINA, N. H.; GENTIL, R. M.; OLIVEIRA, M. B.; SARTORI, M. F.; CABRAL, J. H.; VASCONCELOS, M. S. \& BARROS, O. M., 1994. Investigação epidemiológica do tracoma em pré-escolares e escolares nos municípios de Franco da Rocha e Francisco Morato - São Paulo, 1989. Arquivos Brasileiros de Oftalmologia, 57:154-158.

MEDINA, N. H.; MASSAINI, M. G.; AZEVEDO, C. L. B.; HARIMA, C.; PRADO, M.; MALUF, S.; MARCUCCI, M.; CALIGARIS, L. S. A. \& MORIMOTO, W. T. M., 1998. Vigilância epidemiológica do tracoma em instituição de ensino na cidade de São Paulo, SP. Revista de Saúde Pública, 32:59-63.

MEDINA, N. H.; OLIVEIRA, M. B.; TOBIN, S.; KILL Jr., G.; MENDONÇA, M. M. E. \& BARROS, O. M., 1992. The prevalence of trachoma in preschool and school children in Olímpia, Guaraci and Cajobi, São Paulo, Brazil. Tropical Medicine and Parasitology, 43:121-123.

MELO-OLIVEIRA, S., 1927. Do Tracoma: Complicações, Conseqüências e Tratamento. Tese de Doutorado, Rio de Janeiro: Faculdade de Medicina, Universidade Federal do Rio de Janeiro.

MOREIRA, A. T. R.; SCARPI, M. J.; OYAMA, C.; PENTEADO, R.; MOREIRA, L. B. \& GUIDUGLI, T., 1993. Prevalência de tracoma no município de Miraselva, Estado do Paraná, Brasil. Arquivos Brasileiros de Oftalmologia, 56:354-358.

NOBREGA, M. J.; BONOMO, P. P. O.; SCARPI, M. J.; GUIDUGLI, T.; CAMPOS, C. E. G.; JULIANO, Y. \& NOVO, N. F., 1993. Prevalência de tracoma em crianças pré-escolares e escolares da periferia da cidade de Joinville, Estado de Santa Catarina, Brasil. Arquivos Brasileiros de Oftalmologia, 56:13-17.
SÃO PAULO, 1994. Resolução S.S. no 60/92 de 17 de fevereiro de 1992, alterada D.O.E. 104 (162). Dispõe sobre a lista de doenças de notificação compulsória. São Paulo: Diário Oficial do Estado de São Paulo, Seção I, p. 21, 30 ago.

SCARPI, M. J.; PLUT, R. C. A. \& ARRUDA, H. O., 1989. Prevalência de tracoma no povoado de Mocambo, Estado do Ceará, Brasil. Arquivos Brasileiros de Oftalmologia, 52:177-179.

SES-SP (Secretaria de Estado da Saúde de São Paulo), 1993. Manual de Vigilância Epidemiológica - Tracoma. Normas e Instruções - 1991. 2a Ed. São Paulo: SES-SP.

THYLEFORS, B.; DAWSON, C. R.; JONES, B. R.; WEST, S. K. \& TAYLOR, H. R., 1987. A simple for the assessment of trachoma and its complications. Bulletin of the World Health Organization, 65:477483.

TIELSH, J. M.; WEST Jr., K. P.; KATZ, J.; KEYVAN-LARIJANI, E.; TIZAZU, T.; SCHWAB, L.; JOHNSON, G. J.; CHIRAMBO, M. C. \& TAYLOR, H. R., 1988. The epidemiology of trachoma in Southern Malawi. American Journal of Tropical Medicine and Hygiene, 38:393-399.

TOLEDO, S. A., 1944. O Combate ao Tracoma no Estado de São Paulo. São Paulo: Editora Síntese.

Recebido em 23 de março de 1999

Versão final reapresentada em 21 de novembro de 2001 Aprovado em 8 de fevereiro de 2002 